\title{
Simulation of Alkane Adsorption in the Aluminophosphate Molecular Sieve $\mathrm{AlPO}_{4}-5$
}

\author{
Thierry Maris, ${ }^{\dagger}$ Thijs J. H. Vlugt, and Berend Smit* \\ Department of Chemical Engineering, University of Amsterdam, Nieuwe Achtergracht 166, \\ 1018 WV Amsterdam, The Netherlands
}

Received: February 24, 1998

\begin{abstract}
Adsorption isotherms of alkanes ranging from methane through pentane in the microporous aluminophosphate $\mathrm{AlPO}_{4}-5$ are calculated using grand canonical monte carlo simulations. These simulations predict a surprisingly complex behavior. For methane and ethane we find at low temperatures a low-density-high-density transition that resembles capillary condensation. At intermediate temperatures a high-density structural transition is observed in which the adsorption increases stepwise from four to six molecules per unit cell for methane and two to four molecules per unit cell for ethane. At room temperature a small inflection point in the isotherms signals this transition. For propane, we find a similar high-density transition, but for butane and pentane this transition is not present.
\end{abstract}

\section{Introduction}

$\mathrm{AlPO}_{4}-5$ is a microporous aluminophosphate having cylindrical pores of diameter $7.3 \AA$. The micropores are not interconnected and form one-dimensional channels parallel to the $c$ crystallographic axis (AFI network). This one-dimensional character of the pore system makes AFI an ideal model system for experimental and theoretical studies of processes occurring in a typical one-dimensional channel structure, like, for example, single-file diffusion of small molecules. ${ }^{1-4}$

Recently, Radhakrishnan and Gubbins ${ }^{5}$ have reported a molecular simulation study of methane adsorption using a simple model of $\mathrm{AlPO}_{4}-5$. These simulations show a phase transition occurring between two different density states. The interesting aspect of their results is that this transition is quasi onedimensional. Radhakrishnan and Gubbins propose that this transition is induced by the interactions with the molecules that are in neighboring channels. For their simulations, they used a simple model in which a zeolite channel is modeled as a structureless cylinder. The zeolite is a hexagonal array of these cylinders. It is an interesting question whether or not a similar quasi one-dimensional phase transition can be found in a more sophisticated model of zeolite in which the crystal structure is taken into account explicitly.

A different type of transition in this zeolite was found by Boutin et al. ${ }^{16}$ and Lachet et al. ${ }^{17}$ at a much higher temperature than the phase transition considered by Radhakrishnan and Gubbins. The simulations of Boutin et al. show a stepped isotherm. This step was attributed to a structural rearrangement of the adsorbed high-density phase. An important conclusion of this work is that this transition was only observed using a model that includes both two-body and three-body dispersion interactions, whereas a model with only two-body LennardJones type potential did not show such a step. Such LennardJones type potential is often used in simulations of adsorption

* Corresponding author: smit@chemeng.chem.uva.nl.

†resent address: Inorganic Chemistry Laboratory I.C.L., University of Oxford, South Parks Road, OX1 3QR Oxford, U.K. in zeolites. ${ }^{8-12}$ This conclusion may have important consequences for these simulations. In this work, we study the adsorption of methane in $\mathrm{AlPO}_{4}-5$ for a different set of parameters of the Lennard-Jones potential to investigate whether this conclusion of Boutin et al. and Lachet et al. is generally valid. In addition, we report grand canonical Monte Carlo simulations of adsorption of other linear hydrocarbons in $\mathrm{AlPO}_{4}-5$ over a wide range of pressures and temperatures.

\section{Model and Simulation Details}

Adsorption isotherms are conveniently computed in the grand canonical ensemble. ${ }^{13}$ In this ensemble, the zeolite is in contact with a bath that fixes both the chemical potential and the temperature.

The simulation box contained 60 hexagonal unit cells $(4 \times$ $3 \times 5, a=b=13.72 \AA$ and $c=8.484 \AA$ ) of the zeolite $\mathrm{AlPO}_{4}-5$ with a total volume of $82983 \AA^{3} .{ }^{14}$ This box corresponds to 12 cylindrical channels. Alkanes molecules are modeled using a united-atom representation; i.e., the $\mathrm{CH}_{4}, \mathrm{CH}_{3}$, and $\mathrm{CH}_{2}$ groups are considered as single interaction centers. ${ }^{15}$ We have used a fixed $\mathrm{C}-\mathrm{C}$ bond length of $1.53 \AA$, and the bond-bending and torsion potentials are taken from refs $16-$ 18. Intermolecular interactions are described by the $12-6$ Lennard-Jones potential, which is truncated at $13.8 \AA$, and the usual tail corrections are added. ${ }^{13,19}$ The parameters for the alkane-alkane interaction are taken from refs 20 and 21 and are shown in Table 1.

The zeolite lattice is assumed to be rigid, and zeolite-alkane interactions are assumed to be dominated by dispersive forces involving mainly oxygen atoms. ${ }^{22,23}$ These interactions are also modeled with 12-6 Lennard-Jones potentials. Calculations of the alkane-zeolite potential were performed using a grid of approximatively $0.14 \AA$ spacing in each direction. Energy and size parameters of the alkane-zeolite potential have been fitted to reproduce the Henry coefficient and the heat of adsorption at zero coverage for various alkanes on the zeolite silicalite, ${ }^{10,11}$ see Table 1. We assume here that these parameters are also suitable for $\mathrm{AlPO}_{4}-5$. Since the interactions are dominated by 
TABLE 1: Lennard-Jones Parameters Used in This Work ${ }^{a}$

\begin{tabular}{lccccc}
\hline & $\epsilon / \mathrm{k}_{\mathrm{B}} /(\mathrm{K})$ & $\sigma /(\AA)$ & & $\epsilon / \mathrm{k}_{\mathrm{B}} /(\mathrm{K})$ & $\sigma /(\AA)$ \\
\hline $\mathrm{CH}_{4}-\mathrm{CH}_{4}$ & 148.0 & 3.73 & $\mathrm{CH}_{4}-\mathrm{O}$ & 96.5 & 3.60 \\
$\mathrm{CH}_{3}-\mathrm{CH}_{3}$ & 98.1 & 3.77 & $\mathrm{CH}_{3}-\mathrm{O}$ & 80.0 & 3.60 \\
$\mathrm{CH}_{2}-\mathrm{CH}_{2}$ & 47.0 & 3.93 & $\mathrm{CH}_{2}-\mathrm{O}$ & 58.0 & 3.60
\end{tabular}

${ }^{a}$ All Lennard-Jones interactions were truncated at $13.8 \AA$, and the usual tail corrections have been applied..$^{13,19}$

oxygen atoms, this may be a reasonable approximation. However, since we do not use optimized parameters for $\mathrm{AlPO}_{4}-$ 5 ,we do not expect that our model is in quantitative agreement with experimental results.

In a grand canonical simulation, it is essential to exchange particles successfully with the reservoir. For ethane and the higher alkanes, we have used the configurational-bias Monte Carlo technique to enhance the number of successful exchanges. ${ }^{13,24}$ In every simulation, the number of trial orientations was equal to six. A simulation consists of 150000 cycles, and the number of trial moves in a cycle is equal to the number of particles with a minimum of 20 trial moves per cycle. Further details on computational aspects can be found in refs 10,11 , 25 , and 26.

\section{Simulation Results}

3.1. Methane. Figure 1 presents several simulated adsorption isotherms of methane in $\mathrm{AlPO}_{4}-5$. Isotherms calculated from 18 to $30 \mathrm{~K}$ show a sharp discontinuous jump. To
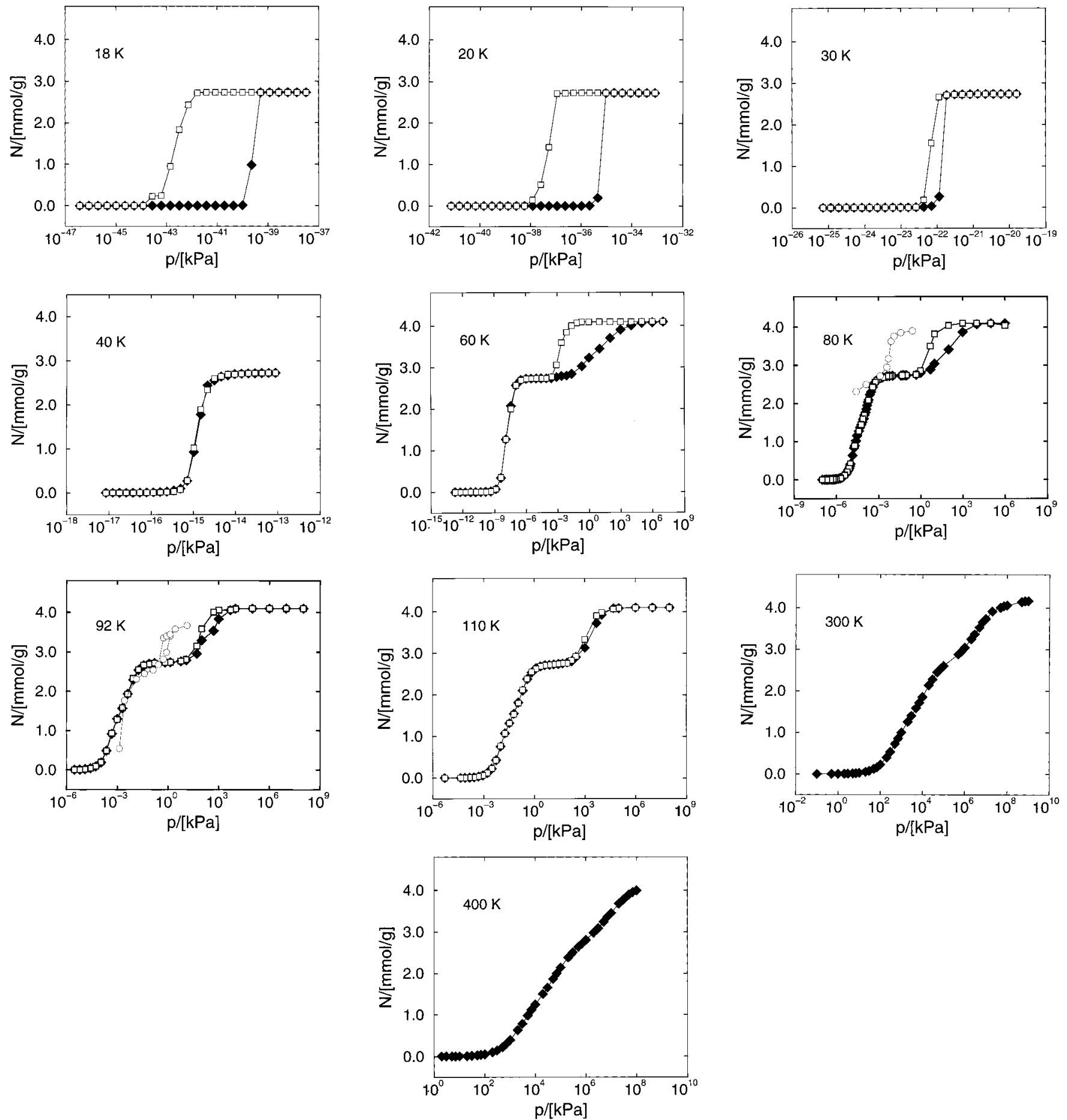

Figure 1. Methane adsorption isotherms on $\mathrm{AlPO}_{4}-5$. The closed symbols are the adsorption branch and the open symbols the desorption branch The circles at 80 and $92 \mathrm{~K}$ are experimental data from ref 30. 
investigate whether hysteresis may occur, we have computed both the isotherms for adsorption and desorption. Simulation of desorption were performed starting from a high-density state. For the isotherms calculated on desorption, the reverse jump occurs at lower chemical potential, giving rise to a hysteresis loop. The hysteresis amplitude decreases when simulation temperature increases, until $33 \mathrm{~K}$ where the simulated isotherm becomes continuous and reversible. Above $60 \mathrm{~K}$, an inflection point in the isotherm is observed when the amount of adsorbed methane reaches four molecules per unit cell (one molecule per unit cell corresponds to $0.68 \mathrm{mmol} / \mathrm{g}$ ). Following this step, the number of adsorbed methane molecules increases until a maximum loading of six methane molecules per unit cell. A hysteresis loop is also found when desorption simulations are performed starting from this last phase. Figure 1 shows that the amplitude of the inflection and the length of the hysteresis decrease with increasing temperature; the hysteresis loop strongly reduces at $92 \mathrm{~K}$ and vanishes at $110 \mathrm{~K}$. At room temperature the step almost disappears.

At low temperatures, we observe a transition that is quite similar to a capillary condensation. This behavior can be related to a first-order phase transition from a gaslike low-density state to a liquidlike high-density phase. We have performed thermodynamics integration following Peterson and Gubbins's method $^{27}$ to make an estimation of the critical temperature. From these calculations it is difficult to estimate the critical point because of the large fluctuations near the critical temperature. From the resulting coexistence phase diagram, the critical temperature can be estimated at $33 \pm 3 \mathrm{~K}$. Note that this temperature is much lower than the critical temperature of methane $(190.4 \mathrm{~K}) .^{28}$

It is interesting to compare our results with those on the simple AFI model of Radhakrishnan and Gubbins. ${ }^{5}$ Radhakrishnan and Gubbins have found different results for isotherms calculated with an isolated pore compared with those obtained using an array of several cylindrical pores. In the first case, the isotherms were Langmuir type I isotherms; hence, no lowdensity vaporlike/high-density liquidlike phase coexistence was observed. For the array of pores Radhakrishnan and Gubbins did observe a low-density-high-density coexistence. It is therefore important to investigate if, in our model of AFI, we restrict the molecules to be in a single pore only, the highdensity-low-density transition also disappears. To make this comparison, we have also performed simulation on a simulation box that consists of $(4 \times 3 \times 50)$ unit cells. In addition, in all pores but one, the potential was set to an infinite value. Hence, the molecules can only adsorb in a single cylinder along the $c$ axis that is 10 times larger as that for the ordinary system.

The simulations with a single pore and the full AFI model gave similar isotherms. For both cases, the phase transition occurs approximatively at the same chemical potential (Figure 2 ). This suggests that correlation effects between adjacent pores are not essential to observe a phase transition when simulations are performed using a more realistic model of $\mathrm{AlPO}_{4}-5$.

An analysis of the first high-density phase (Figure 3) shows that this state corresponds to a loading of four adsorbed methane molecules per AFI unit cell. The distribution along one channel is not continuous, the adsorbed molecules are located exclusively at $c / 4$ and $3 c / 4$, where the adsorption sites are located (six sites at $c / 4$ and six at $3 c / 4$ [7]). At $c / 4$ and $3 c / 4$ we observe two methane molecules, and we can see in Figure 3 that these adsorbed molecules are placed following a helix arrangement. The centers of the pores are never occupied.

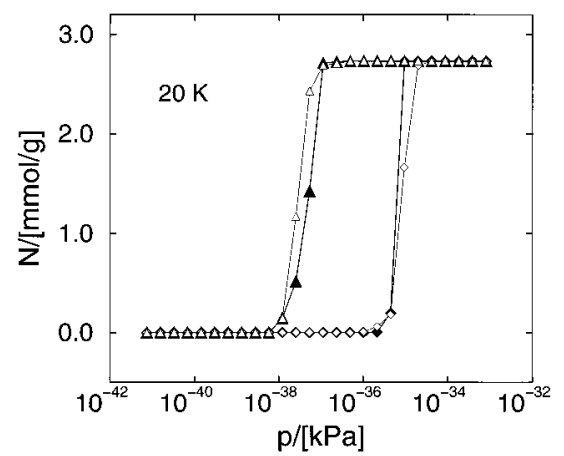

Figure 2. Methane adsorption isotherms on $\mathrm{AlPO}_{4}-5$ calculated at $20 \mathrm{~K}$. Closed symbols and open symbols are isotherms calculated with 12 and 1 channels, respectively. Triangles are the adsorption branch and diamonds the desorption branch.
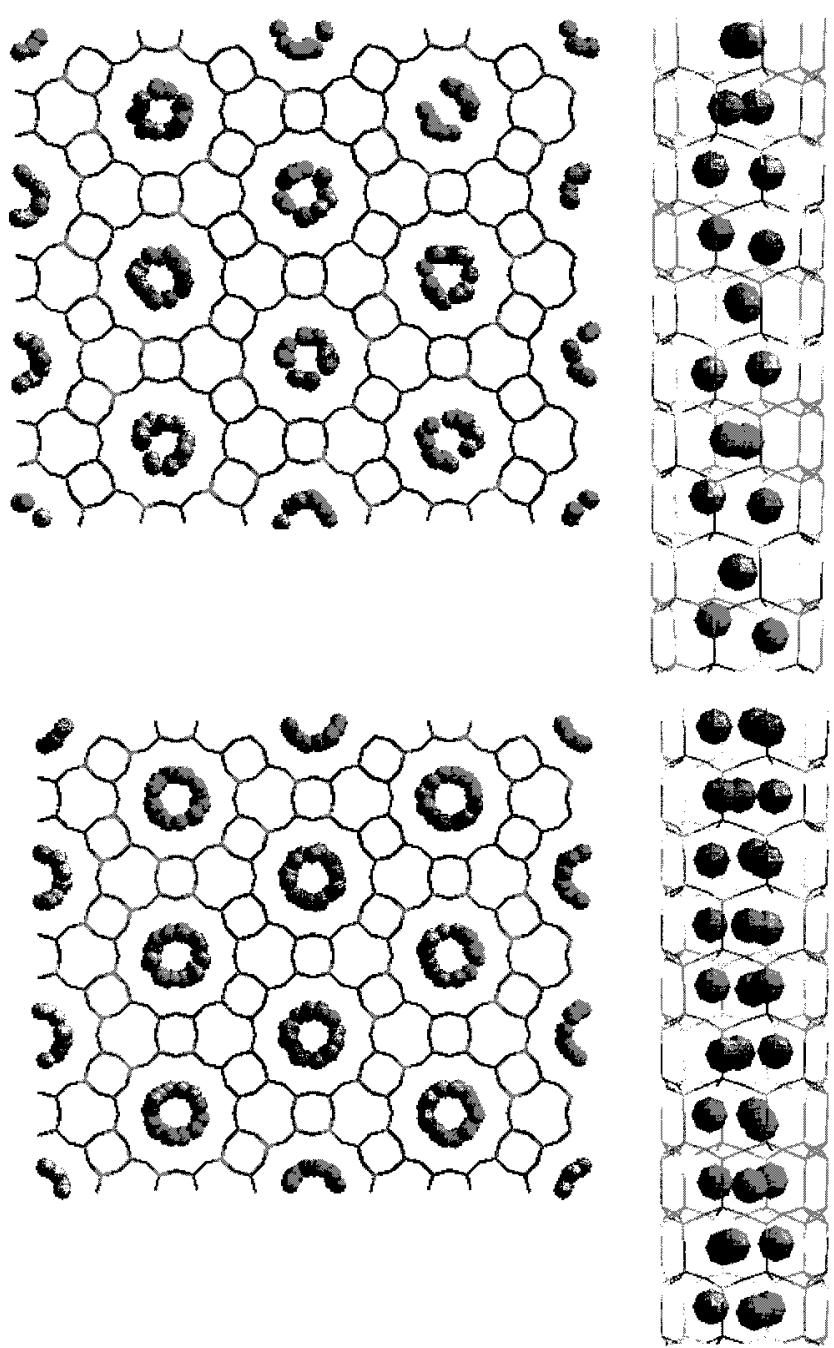

Figure 3. Snapshots of the different methane adsorbed phases. The lines represent the zeolite structure and the spheres the methane molecules. The top figure is the first high-density state with four molecules per unit cell, and the bottom figure is the second high-density state with six molecules per unit cell. The left figures are a projection on the $(a, b)$ plane, and the right figures a view of one channel on the $(a, c)$ plane.

The simulations at higher temperature show that increasing the pressure results in a new condensed state, with six adsorbed methane molecules per unit cell, which are located at $c / 4$ and $3 c / 4$ (Figure 3). The resulting structure forms also a layered arrangement. This phase has already been described as the $\{0-$ 3-0-3\} structure type found by Lachet et al. from simulations 

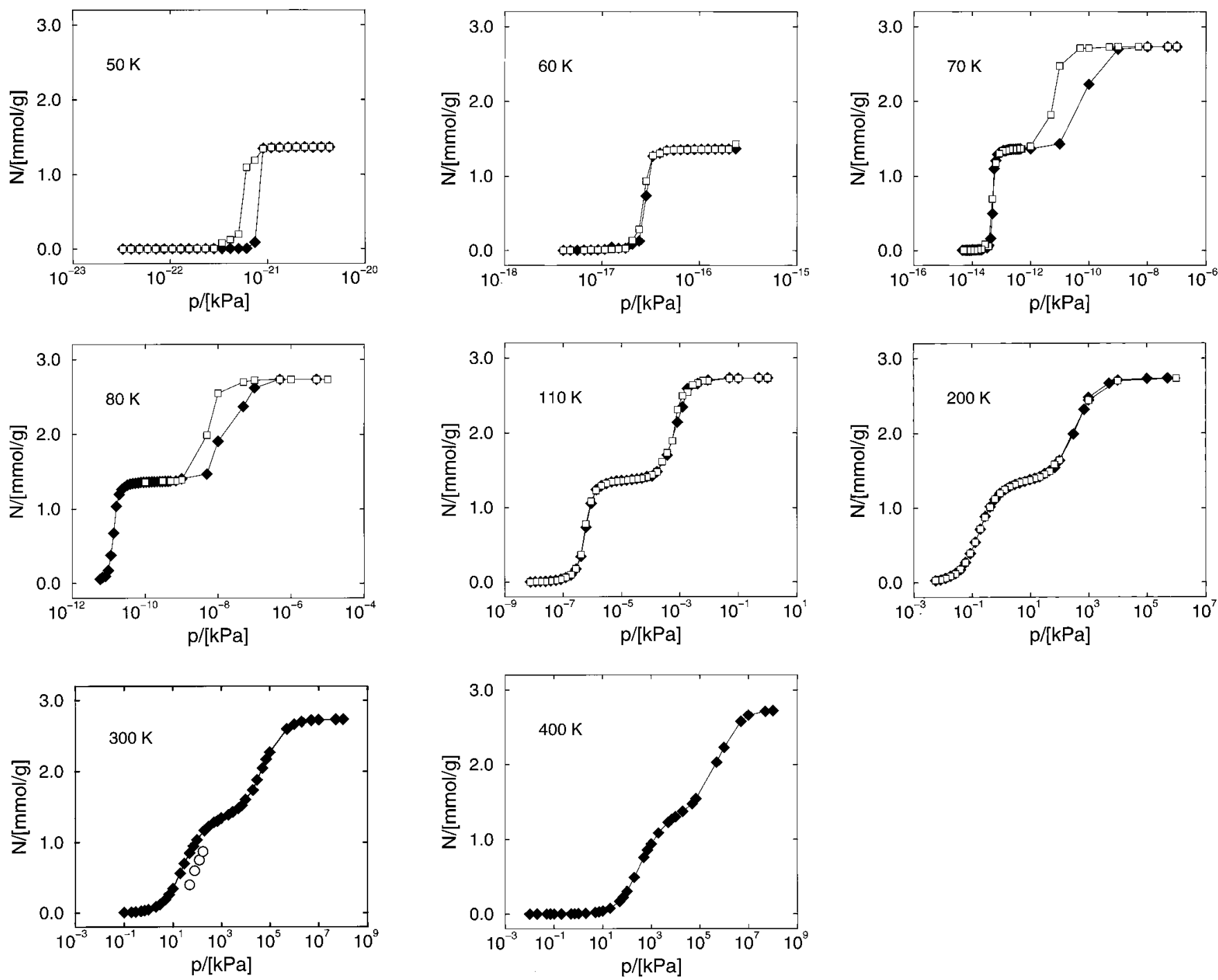

Figure 4. Ethane adsorption isotherms on $\mathrm{AlPO}_{4}-5$. The closed symbols are the adsorption branch and the open symbols the desorption branch. The open circles at $300 \mathrm{~K}$ are experimental data from ref 34 .

involving their M3 potential. ${ }^{7}$ Whereas Boutin et al. ${ }^{6}$ and Lachet et al. concluded that three-body interactions are essential, our simulations show that the classical two-body Lennard-Jones type potential is sufficient to reproduce the stepped isotherms and the maximum loading with six methane molecules per unit cell. It is important to note that we have used a different set of Lennard-Jones parameters compared to those used in refs 6 and 7. A calculation at $77.3 \mathrm{~K}$ with the Lennard-Jones methanezeolite parameters used by Lachet et al. $\left(\epsilon / k_{\mathrm{B}}=100.0 \mathrm{~K}\right.$ and $\sigma$ $=3.233 \AA$ ) and the methane-methane potential from Table 1 gives similar qualitative results, which implies that the difference in the adsorption isotherm shape cannot be explained only on the basis of the difference in the Lennard-Jones parameter for the methane-zeolite interaction. A possible explanation may be the difference in the grid size and the interpolation procedure used to calculate zeolite-alkane interactions. We have used a grid spacing of $0.14 \AA$, whereas in refs 6 and 7, a spacing of approximative $0.2 \AA$ is used. Another explanation might be the slightly different methane-methane potential in the work of Lachet et al.

It is interesting to compare our data with the experimental results of Martin et al., ${ }^{29,30}$ see Figure 1. The shape of the experimental coexistence curves at $T=77.3 \mathrm{~K}$ and $T=96.5$ $\mathrm{K}$ is very similar to ours; both studies observe a step in the adsorption isotherm. As can be expected the quantitative agreement is not as good since our parameters have not been optimized for $\mathrm{AlPO}_{4}-5$. It is interesting to note that the shape of the hysteresis curve as found in the experiments is very similar to ours. For $T=77.3 \mathrm{~K}$ it was not possible to measure hysteresis. In addition, our simulations confirm that this experimentally observed hysteresis loop is not related to capillary condensation.

The mechanism of hysteresis has been the subject of many investigations. ${ }^{31}$ Statistical mechanical theories assume that this hysteresis arises from a metastable state in a single pore. Other theories, however, assume that the way a pore is filled or emptied (due to instabilities of adsorbed films) has an influence on the shape of the hysteresis curve. ${ }^{32,33}$ This implies that we have to be careful to compare the shape of the simulated hysteresis curve with the experiments. Furthermore, we have to realize that if we would perform infinitely long simulations of an infinitely large system we would not observe any hysteresis at all. However, a finite system in a metastable state is relatively stable to small perturbations. In a Monte Carlo simulation, trial moves that introduce large perturbations (for example, filling or emptying the zeolite completely in one trial move) are never performed because in practice they will never be accepted. It is essential that in our simulations we increased the chemical potential in small steps and started from a previous configuration. The fact that we observe a hysteresis that is similar in 

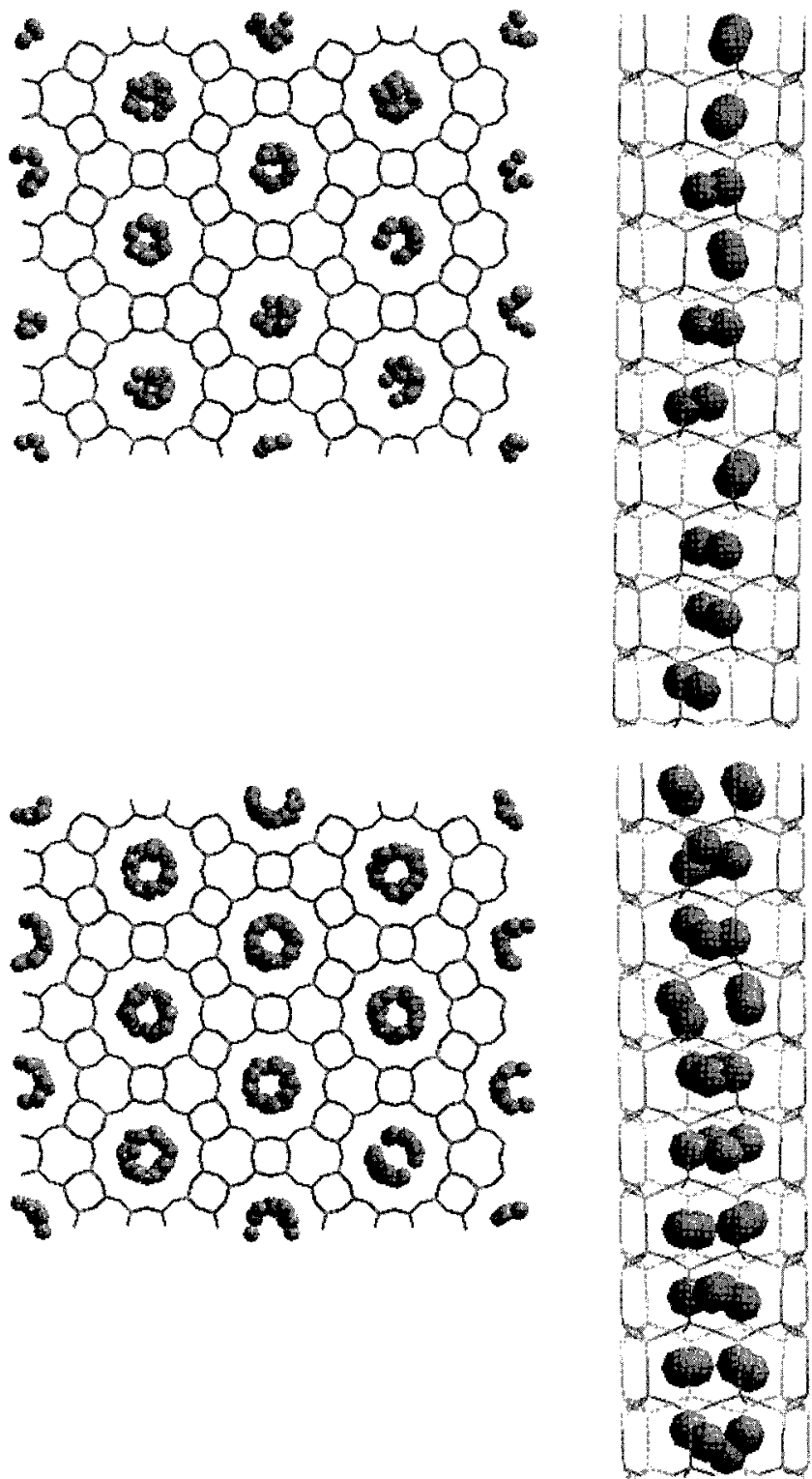

Figure 5. Snapshots of the different ethane adsorbed phases. The lines represent the zeolite structure and the spheres the atoms of the ethane molecules. The top figure is the first high-density state with two molecules per unit cell and the bottom figure the second high-density state with four molecules per unit cell. The left figures are a projection on the $(a, b)$ plane and the right figures a view of one channel on the $(a, c)$ plane.

shape to the experimental one may be some evidence that the mechanism for this case is the metastable states.

3.2. Ethane. Ethane adsorption on $\mathrm{AlPO}_{4}-5$ is similar to the behavior of methane. At low temperatures, we observe a
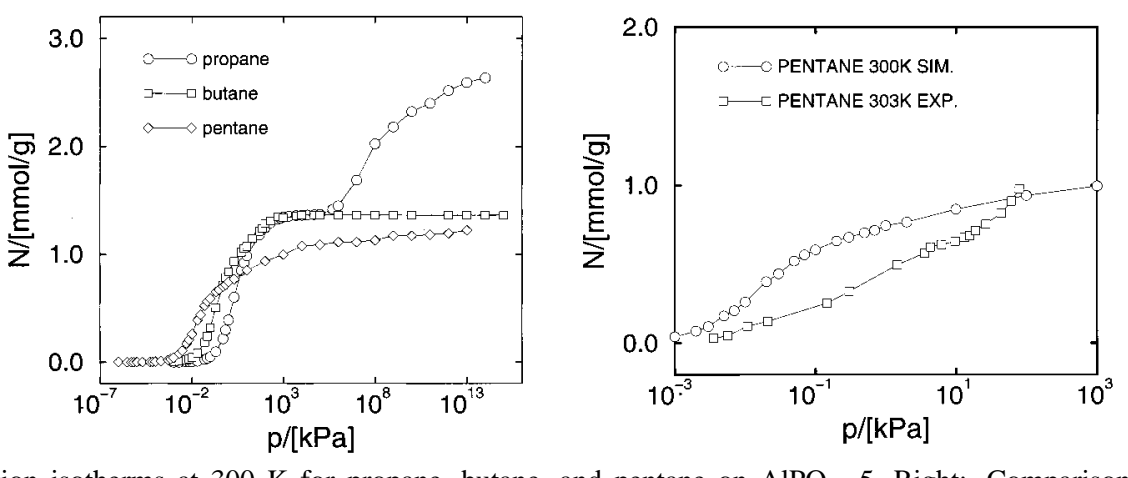

Figure 6. Left: Adsorption isotherms at $300 \mathrm{~K}$ for propane, butane, and pentane on $\mathrm{AlPO}_{4}-5$. Right: Comparison between simulations for pentane at $300 \mathrm{~K}$ with experimental data from ref 4 at $303 \mathrm{~K}$.

low-density vaporlike-high-density liquidlike transition with a critical point $T_{\mathrm{c}}=55 \pm 3 \mathrm{~K}$ (Figure 4), which is much lower than the critical temperature of ethane $(305.4 \mathrm{~K}){ }^{28}$ The concentration of the high-density state is two ethane molecules per hexagonal AFI unit cell. At high temperature, an inflection in the isotherm is also displayed in much the same way as observed for methane. For ethane, the maximum loading is four adsorbed molecules per unit cell. The simulations shows a large hysteresis loop at 70 and $80 \mathrm{~K}$. The length of the step decreases with increasing temperature, and, at 300 and $400 \mathrm{~K}$, only an inflection point can be observed. At $300 \mathrm{~K}$, we have also plotted experimental data from ref 34 at $305 \mathrm{~K}$. The agreement is reasonable.

In the first high-density state, two ethane molecules per unit cell are adsorbed at $c / 4$ and $3 c / 4$. The $(a, b)$ projection of Figure 5 shows that the center of the pores remains unoccupied. When all the sites are occupied, a large driving force is needed to add an additional ethane molecule in each layer, which results in a large inflection at low temperatures. When the pressure is sufficiently high, additional molecules can adsorb resulting in the second high-density phase with four ethane molecules per unit cell.

3.3. Propane, Butane, and Pentane. For short-chain alkanes, the adsorbed molecules are trapped in specific adsorption sites and are not distributed continuously along the channels. The adsorption sites are limited in size, and one should therefore expect that, for longer alkanes, the selective adsorption on these sites become more difficult. To investigate how the longer alkanes are adsorbed in the channels, we have calculated the isotherms for propane, butane, and pentane at $300 \mathrm{~K}$. These isotherms are shown Figure 6. We have compared our simulations of pentane with experimental data from ref 4 . Because our alkane-zeolite parameters were optimized for silicalite, we may expect some differences. However, the agreement is reasonable.

The simulated adsorption isotherm for propane at $300 \mathrm{~K}$ shows also a step when the loading is close to two molecules per unit cell. Figure 7 shows a snapshot of the adsorbed phase at the loading where the isotherm reaches the step. Figure 7 also shows the probability distribution of propane at maximum loading. Also propane shows a preferential adsorption at $c / 4$ and $3 c / 4$, but the probability to find molecules between these sites is higher than for methane and ethane. The snapshot at maximum loading shows that it is still possible to put two propane molecules at $c / 4$ and $3 c / 4$, but the propane molecules can also be located out from these specific positions. For butane and pentane, the insertion needs a high pressure and the maximum loading is lower compared to those observed for propane. The probability distribution in Figure 8 shows that molecules can move freely through the entire channel and are 

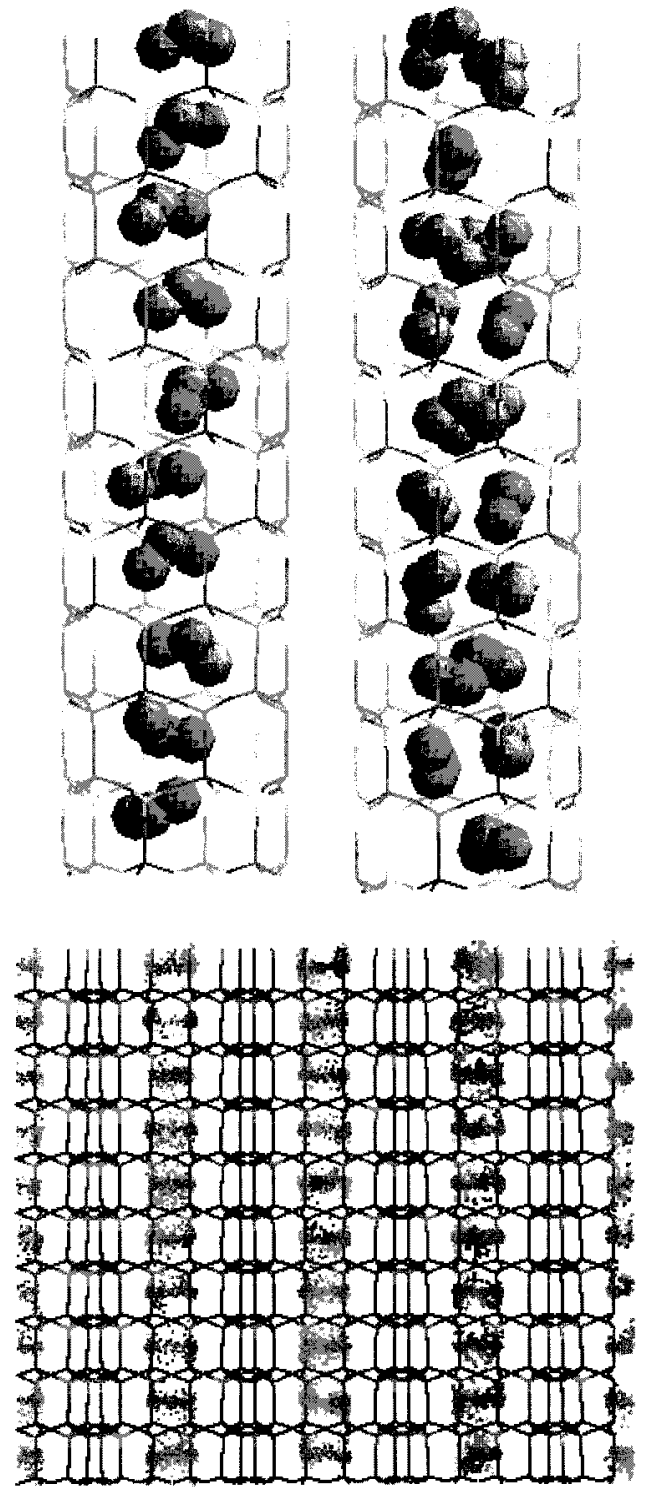

Figure 7. Snapshot of the propane adsorbed phase. The top left figure is a projection on the $(a, c)$ plane of the high-density state observed in the step of the isotherms. The top right figure is the same projection for the phase at very high pressure. The bottom figure represents a probability distribution of the high-pressure propane adsorbed phase. At regular intervals during the simulation, the positions of the center of mass of the molecules are computed and plotted. The density of the dots represents a measure of the probability of finding the alkane molecule in the different parts of the zeolite.

not trapped near the adsorption sites. It seems that pentane can occupy the center of the pore where there is no adsorption site (compare Figures 3 and 8). In this configuration, both tails of pentane occupy adsorption sites at $c / 4$ and $3 c / 4$. The entire channel can be packed by these molecules, and no step in the adsorption isotherm is observed.

\section{Concluding Remarks}

The adsorption of short-chain alkanes in $\mathrm{AlPO}_{4}-5$ has a surprisingly complex behavior. The complex behavior of methane in $\mathrm{AlPO}_{4}-5$, as observed experimentally, can be reproduced qualitatively using a simple two-body Lennard-Jones potential. In this study we have used parameters that were optimized for all silica structures. As can be expected, to obtain a better quantitative agreement with experiments these parameters should be optimized for aluminophosphates.
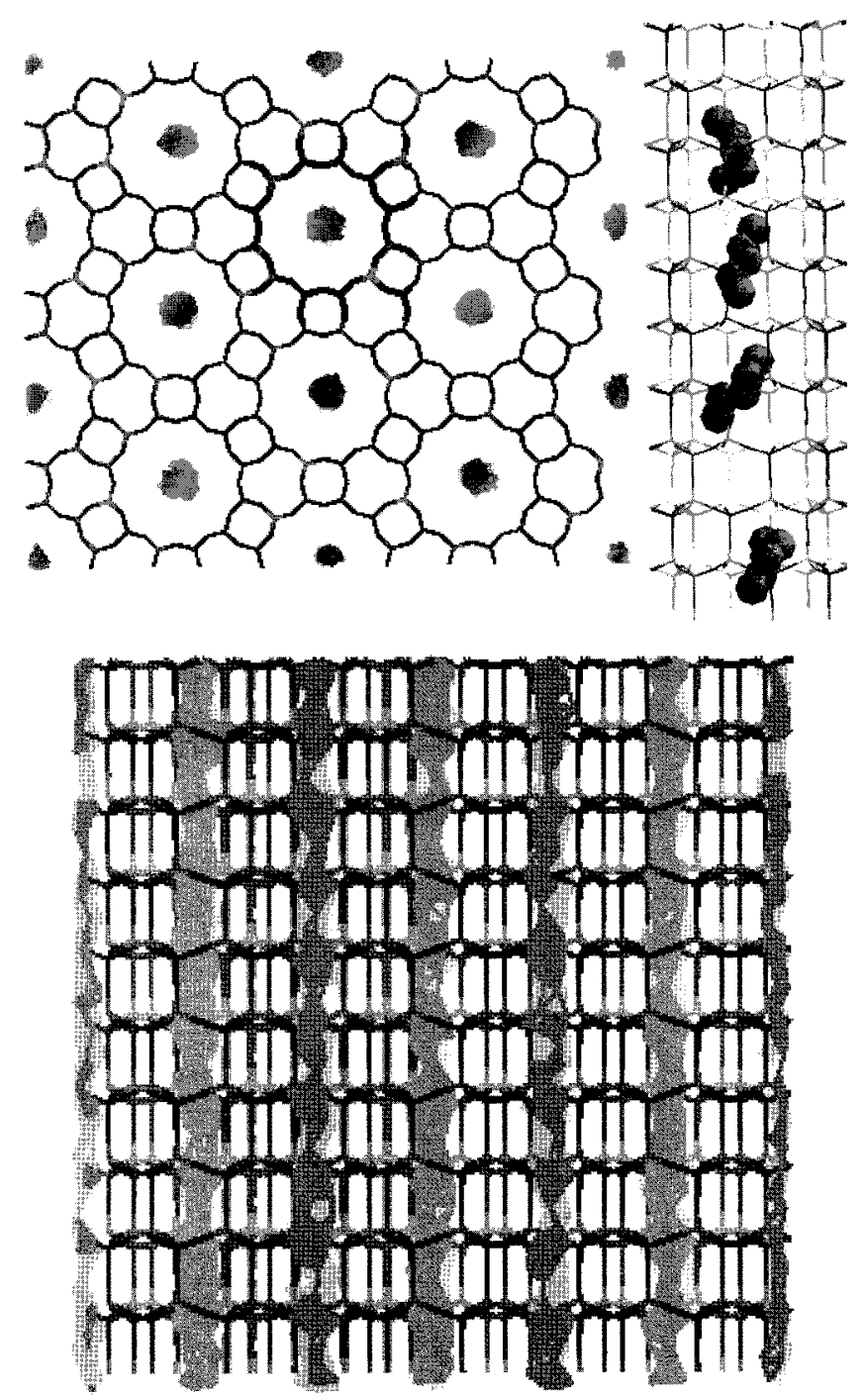

Figure 8. Probability distribution of the high-pressure pentane adsorbed phase. The top figure is a projection on the $(a, b)$ plane and the bottom figure a projection on the $(a, c)$ plane. The right top figure represents a snapshot of one channel on the $(a, c)$ plane.

Acknowledgment. Financial support provided to T.J.H.V. from SON (Stichting Scheikundig Onderzoek Nederland) and to B.S. from the OSPT are acknowledged. A large part of the computer resources were generously provided by SARA (Stichting Academisch Rekencentrum Amsterdam).

\section{References and Notes}

(1) Nivarthi, S.; McCormick, A. V.; Davis, H. T. Chem. Phys. Lett. 1994, 229, 297-301.

(2) Gupta, S.; Nivarthi, S. S.; McCormick, A. V.; Danish, H. T. Chem. Phys. Lett. 1995, 247, 596-600.

(3) Keffer, D.; McCormick, A., V.; Davis, T. H. Mol. Phys. 1996, 87, 367-387.

(4) Newalkar, B. L.; Jasra, R. V.; Kamath, V.; Bhat, S. G. T. Microporous Mater. 1997, 11, 195-205. 2850 .

(5) Radhkrishnan, R.; Gubbins, E. Phys. Rev. Lett. 1997, 79, 2847-

(6) Boutin, A.; Pellenq, R. J. M.; Nicholson, D. Chem. Phys. Lett. 1994, 219, 484-490.

(7) Lachet, V.; Boutin, A.; Pellenq, R. J. M.; Nicholson, D.; Fuchs, A. H. J. Phys. Chem. 1996, 100, 9006-9013.

(8) Smith, B. Mol. Phys. 1995, 85, 153-172.

(9) Smit, B.; Maesen, T. L. M. Nature 1995, 374, 42-44.

(10) Du, Z.; Vlugt, T. J. H.; Smit, B.; Manos, G. AlChE J. 1998, 44, $1756-1764$

(11) Vlugt, T. J. H.; Zhu, W.; Moulijn, J. A.; Smit, B., Krishna, R. J. Am. Chem. Soc. 1998, 120, 5599-5600. 
(12) Bates, S. P.; van Well, W. J. M.; van Santen, R. A.; Smit, B. J. Am. Chem. Soc. 1996, 118, 6753-6759.

(13) Frenkel, D.; Smit, B. Understanding Molecular Simulations: from Algorithms to Applications; Academic Press: San Diego, CA, 1996.

(14) Bennett, J. M.; Cohen, J. P..; Flanigen, E. M.; Pluth, J. J.; Smit, J. V. ACS Symp. 1983, 208, 109.

(15) Martin, M. G.; Siepmann, J. I. J. Phys. Chem. 1998, 102, 25692577.

(16) Van der Ploeg, P.; Berendsen, H. J. C. J. Chem. Phys. 1982, 76, $3271-3276$

(17) Ryckaert, J. P.; Bellemans, A. Faraday Discuss. Chem. Soc. 1978, $66,95-106$

(18) Wang, Y.; Hill, K.; Harris, J. G. J. Phys. Chem. 1994, 100, 32763285 .

(19) Allen, M. P.; Tildesley, D. J. Computer Simulation of Liquids Clarendon Press: Oxford, 1987.

(20) Siepmann, I. J.; Martin, M. G.; Mundy, C. J.; Klein, M. L. Mol. Phys. 1997, 90, 687-693.

(21) Verlet, L.; Weis, J. J. Mol. Phys. 1972, 24, 1013-1024.

(22) Bezus, A. G.; Kiselev, A. V.; Lopatkin, A. A.; Du, P. Q. J. Chem. Soc., Faraday Trans. 2 1978, 74, 367-379.

(23) Kiselev, A. V.; Lopatkin, A. A.; Shulga, A. A. Zeolites 1985, 5 , $261-267$.
(24) Smit, B. J. Phys. Chem. 1995, 99, 5597-5603.

(25) Vlugt, T. J. H.; Martin, M. G.; Smit, B.; Siepmann, J. I.; Krishna, R. Mol. Phys. 1998, 94, 727-733.

(26) Esselink, K.; Loyens, L. D. J. C.; Smit, B. Phys. Rev. E 1995, 51, $1560-1568$.

(27) Peterson, B. K.; Gubbins, K. E. Mol. Phys. 1987, 62, 215-226.

(28) Reid, R. C.; Prausnitz, J. M.; Poling, B. E. The Properties of Gases and Liquids; McGraw-Hill: New York, 1987.

(29) Martin, C.; Coulomb, J. P.; Grillet, Y.; Kahn, R. In Fundamentals of Adsorption; Proceedings of the Fifth International Conference; LeVan, M. D., Ed.; Kluwer Academic Publishers: Boston, MA, 1996; p 587.

(30) Martin, C.; Tosi-Pellenq, N.; Patarin, J.; Coulomb, J. P. Langmuir 1998, 14, 1774-1778.

(31) Evans, R. In Liquides aux Interfaces/Liquids at interfaces; Les Houches, Session XLVIII; 1988; Charvolin, J.; Joanny, J. F.; Zinn-Justin, J., Eds.; North Holland: Amsterdam, 1990; pp 1-98.

(32) Marconi, U. M. B.; van. Swol, F. Europhys. Lett. 1989, 9, 531535

(33) Ball, P. C.; Evans, R. Europhys. Lett. 1987, 4, 715-721.

(34) Choudhary, V. R.; Mayadevi, S. Langmuir 1996, 12, 980-985. 